Rinaldi Sabina (Orcid ID: 0000-0002-6846-1204)

Dashti Seyedeh Ghazaleh (Orcid ID: 0000-0002-1399-7220)

Kvaskoff Marina (Orcid ID: 0000-0002-4557-3772)

Ricceri Fulvio (Orcid ID: 0000-0001-8749-9737)

Tsilidis Konstantinos (Orcid ID: 0000-0002-8452-8472)

\title{
Mediation analysis of the alcohol-postmenopausal breast cancer relationship by sex hormones in the EPIC Cohort
}

Nada Assi ${ }^{1}$, Sabina Rinaldi ${ }^{2}$, Vivian Viallon ${ }^{1}$, S. Ghazaleh Dashti ${ }^{1,3}$, Laure Dossus ${ }^{1}$, Agnès Fournier ${ }^{4,5,36}$, Iris Cervenka ${ }^{4,5}$, Marina Kvaskoff ${ }^{4,5}$, Renée Turzanski-Fortner ${ }^{6}$, Manuela Bergmann ${ }^{7}$, Heiner Boeing ${ }^{7}$, Salvatore Panico ${ }^{8}$, Fulvio Ricceri ${ }^{9,10}$, Domenico Palli ${ }^{11}$, Rosario Tumino ${ }^{12}$, Sara Grioni ${ }^{13}$, María José Sánchez Pérez ${ }^{14,15}$, María-Dolores Chirlaque 14,16,17 , Catalina Bonet ${ }^{18}$, Aurelio Barricarte Gurrea ${ }^{14,19,20}$, Pilar Amiano Etxezarreta ${ }^{14,21}$, Susana Merino ${ }^{22}$, Bas Bueno de Mesquita ${ }^{23,24,25,26}$, Carla H. van Gils ${ }^{27}$, Charlotte OnlandMoret ${ }^{27}$, Anne Tjønneland ${ }^{28,29}$, Kim Overvad ${ }^{30}$, Antonia Trichopoulou ${ }^{31}$, Georgia Martimianaki ${ }^{31}$, Anna Karakatsani ${ }^{31,32}$, Tim Key ${ }^{33}$, Sofia Chistakoudi ${ }^{25,34}$, Merete Ellingjord-Dale ${ }^{25}$, Kostas Tsilidis ${ }^{25,35}$, Elio Riboli ${ }^{25}$, Rudolf Kaaks ${ }^{6}$, Marc J Gunter ${ }^{36}$, Pietro Ferrari ${ }^{1^{*}}$

\footnotetext{
Affiliation:

${ }^{1}$ Nutritional Methodology and Biostatistics Group, International Agency for Research on Cancer (IARC), 150 cours Albert Thomas, 69008 Lyon, France.

${ }^{2}$ Biomarkers Group, International Agency for Research on Cancer (IARC), 150 cours Albert Thomas, 69008 Lyon, France.

${ }^{3}$ Center for Epidemiology and Biostatistics, The University of Melbourne School of Population and Global Health, Parkville, VIC, Australia.

${ }^{4}$ CESP, Fac. de médecine - Univ. Paris-Sud, Fac. de médecine - UVSQ, INSERM, Université Paris-Saclay, 94805, Villejuif, France.

${ }^{5}$ Gustave Roussy, F-94805, Villejuif, France.

${ }^{6}$ Division of Cancer Epidemiology, German Cancer Research Center (DKFZ), Heidelberg, Germany.

${ }^{7}$ Department of Epidemiology, German Institute of Human Nutrition, Potsdam-Rehbruecke, 14558 Nuthetal, Germany.

${ }^{8}$ Dipartimento di Medicina Clinica e Chirurgia, Federico II University, Naples, Italy.

${ }^{9}$ Department of Clinical and Biological Sciences, University of Turin, Italy.

${ }^{10}$ Unit of Epidemiology, Regional Health Service ASL TO3, Grugliasco (TO), Italy.

${ }^{11}$ Cancer Risk Factors and Life-Style Epidemiology Unit, Institute for Cancer Research, Prevention and Clinical Network - ISPRO, Florence, Italy.

${ }^{12}$ Cancer Registry and Histopathology Department, “Civic -M.P.Arezzo” Hospital, ASP Ragusa, Italy.

${ }^{13}$ Epidemiology and Prevention Unit, Fondazione IRCCS Istituto Nazionale dei Tumori di Milano, Via Venezian 1, 20133, Milan, Italy.

${ }^{14}$ CIBER de Epidemiología y Salud Pública (CIBERESP), Madrid, Spain.
}

This is the author manuscript accepted for publication and has undergone full peer review but has not been through the copyediting, typesetting, pagination and proofreading process, which may lead to differences between this version and the Version of Record. Please cite this article as doi: $10.1002 / \mathrm{ijc} .32324$

This article is protected by copyright. All rights reserved. 
15 Escuela Andaluza de Salud Pública. Instituto de Investigación Biosanitaria, IBS GRANADA, Universidad de Granada. Granada, Spain.

${ }^{16}$ Department of Epidemiology, Regional Health Council, IMIB-Arrixaca, Murcia, Spain.

${ }^{17}$ Department of Health and Social Sciences, Universidad de Murcia, Murcia, Spain.

${ }^{18}$ Unit of Nutrition and Cancer, Cancer Epidemiology Research Program, Institut Català d’Oncologia, Av. Granvia de L’Hospitalet 199-203, 08908 L’Hospitalet de Llobregat, Spain.

${ }^{19}$ Navarra Public Health Institute, Pamplona, Spain.

${ }^{20}$ Navarra Institute for Health Research (IdiSNA) Pamplona, Spain.

${ }^{21}$ Public Health Division of Gipuzkoa, BioDonostia Research Institue, San Sebastian, Spain.

${ }^{22}$ Public Health Directorate, Asturias, Spain.

${ }^{23}$ Department. for Determinants of Chronic Diseases (DCD), National Institute for Public Health and the Environment (RIVM), PO Box 1, 3720 BA Bilthoven, The Netherlands.

${ }^{24}$ Department of Gastroenterology and Hepatology, University Medical Centre, Utrecht, The Netherlands.

${ }^{25}$ Department of Epidemiology and Biostatistics, MRC-HPA Centre for Environment and Health, School of Public Health, Imperial College London, Norfolk Place W2 1PG London, UK.

${ }^{26}$ Department of Social \& Preventive Medicine, Faculty of Medicine, University of Malaya, Pantai Valley, 50603, Kuala Lumpur, Malaysia.

${ }^{27}$ Julius Center for Health Sciences and Primary Care, Cancer Epidemiology University Medical Center Utrecht, 3508 GA Utrecht, The Netherlands.

${ }^{28}$ Danish Cancer Society Research Center, Strandboulevarden 49, DK 2100 Copenhagen, Denmark.

${ }^{29}$ Department of Public Health, Faculty of Health and Medical Sciences, University of Copenhagen, Denmark.

${ }^{30}$ The Department of Epidemiology, School of Public Health, Aarhus University, Aarhus, Denmark.

${ }^{31}$ Hellenic Health Foundation, Athens, Greece.

${ }^{32}$ Pulmonary Medicine Department, School of Medicine, National and Kapodistrian University of Athens, “ATTIKON” University Hospital, Haidari, Greece.

${ }^{33}$ Cancer Epidemiology Unit, University of Oxford, Oxford, United Kingdom.

${ }^{34}$ MRC Centre for Transplantation, King's College London, Great Maze Pond, London SE1 9RT, United Kingdom.

${ }^{35}$ Department of Hygiene and Epidemiology, University of Ioannina School of Medicine, Ioannina, Greece.

${ }^{36}$ Nutritional Epidemiology Group, International Agency for Research on Cancer (IARC), 150 cours Albert Thomas, 69008 Lyon, France.

\section{Running Title}

Mediation by sex hormones of the alcohol and breast cancer association 
*Corresponding author: Pietro Ferrari, International Agency for Research on Cancer, 150 Cours Albert Thomas, 69372 Lyon cedex 08, France. Tel: +33 47273 8031; Fax: +33 47273 8361. E-mail: ferrarip@iarc.fr

\section{List of abbreviations used}

1-SD: one standard deviation; 95\%CI: 95\% Confidence Intervals; AICR: American Institute of Cancer Research; BC: Breast Cancer; BMI: Body Mass Index; DHEAS:

dehydroepiandrosterone sulfate; DKFZ: German Cancer Research Center; EPIC: European Prospective Investigation into Cancer and nutrition; ER(+ or -): Estrogen Receptor (positive or negative); IARC: International Agency for Research on Cancer; ICD-10: International Classification of Diseases 10th Revision; NDE: Natural Direct Effect; NIE: Natural Indirect Effect; OR: Odds ratio; PLS: Partial Least Squares; RD: Risk Difference; SHBG: Sex Hormone Binding Globulin; TE: Total Effect; WCRF: World Cancer Research Fund.

Word count: 3,949 after revision.

Table count: 4 tables and 4 supplemental tables after revision.

\section{Conflict of Interests Statement}

The authors declare no potential conflicts of interest.

\section{Sources of Support:}

N. Assi was financially supported by a grant from the Fondation de France (FdF) supporting her postdoctoral research (grant number: 00069254). T. Key was funded through a grant from Cancer Research UK (grant number: CRUK C8221/A19170). The steroids measurements used in this study were (partly) funded by a grant from the German Research Foundation, Graduiertenkolleg 793: Epidemiology of communicable and chronic non-communicable

This article is protected by copyright. All rights reserved. 
diseases and their interrelationships and through financial support from the National Cancer Institute (USA) (grant no. 1U01CA98216-01). The coordination of EPIC is financially supported by the European Commission (DG-SANCO) and the International Agency for Research on Cancer. The national cohorts are supported by Danish Cancer Society (Denmark); Ligue Contre le Cancer, Institut Gustave Roussy, Mutuelle Générale de l’Education Nationale, Institut National de la Santé et de la Recherche Médicale (INSERM) (France); Deutsche Krebshilfe, Deutsches Krebsforschungszentrum and Federal Ministry of Education and Research (Germany); the Hellenic Health Foundation (Greece); Associazione Italiana per la Ricerca sul Cancro-AIRC-Italy and National Research Council (Italy); Dutch Ministry of Public Health, Welfare and Sports (VWS), Netherlands Cancer Registry (NKR), LK Research Funds, Dutch Prevention Funds, Dutch ZON (Zorg Onderzoek Nederland), World Cancer Research Fund (WCRF), Statistics Netherlands (The Netherlands); Nordic Centre of Excellence programme on Food, Nutrition and Health. (Norway); Health Research Fund (FIS), PI13/00061 to Granada), Regional Governments of Andalucía, Asturias, Basque Country, Murcia (no. 6236) and Navarra, ISCIII RETIC (RD06/0020) (Spain); Swedish Cancer Society, Swedish Scientific Council and County Councils of Skåne and Västerbotten (Sweden); Cancer Research UK (14136 to EPIC-Norfolk; C570/A16491 and C8221/A19170 to EPIC-Oxford), Medical Research Council (1000143 to EPIC-Norfolk and MR/M012190/1 to EPIC-Oxford) (United Kingdom).

This study was done independently and with no input from the funders. The funders were not involved in the design, implementation, analysis or interpretation of the data. 
"For information on how to submit an application for gaining access to EPIC data and/or biospecimens, please follow the instructions at http://epic.iarc.fr/access/index.php"

What's new? (74 words - limit: 75)

We examined whether sex hormones and SHBG, individually or through a composite hormonal signature, act as mediators on the pathway between alcohol intake and postmenopausal BC. While limited evidence suggested a mediated proportion of $19 \%$ of the total effect through free estradiol, mediation by individual sex-hormone levels suggested a borderline significant indirect effect through free estradiol accounting for $19 \%$ of the mediated proportion, However, the hormonal signature mediated about $24 \%$ of the alcoholBC association, suggesting that any potential mechanism of sex-steroids in the alcohol and BC relationship is likely to involve interplay of hormones, beyond the action of single hormonal levels.

This article is protected by copyright. All rights reserved. 
Abstract (250 words unstructured)

Alcohol consumption is associated with higher risk of breast cancer (BC); however, the biological mechanisms underlying this association are not fully elucidated, particularly the extent to which this relationship is mediated by sex hormone levels.

Circulating concentrations of estradiol, testosterone, their free fractions and sex-hormone binding globulin (SHBG), were examined in 430 incident BC cases and 645 matched controls among alcohol-consuming postmenopausal women nested within the European Prospective Investigation into Cancer and Nutrition. Mediation analysis was applied to assess whether individual hormone levels mediated the relationship between alcohol intake and BC risk. An alcohol-related hormonal signature, obtained by Partial Least Square (PLS) regression, was evaluated as a potential mediator. Total (TE), natural direct (NDE) and natural indirect effects (NIE) were estimated.

Alcohol intake was positively associated with overall BC risk and specifically with estrogen receptor positive tumours with respectively TE=1.17(95\%CI: 1.01,1.35) and 1.36(1.08,1.70) for a 1-SD deviation increase of intake. There was no evidence of mediation by sex steroids or SHBG separately except for a weak indirect effect through free estradiol where NIE=1.03(1.00,1.06). However, an alcohol-related hormonal signature negatively associated with SHBG and positively with estradiol and testosterone, was associated with BC risk (OR=1.25 $(1.07,1.47))$ for a 1-SD higher PLS score, and had a statistically significant NIE accounting for a mediated proportion of $24 \%$.

This article is protected by copyright. All rights reserved. 
There was limited evidence of mediation of the alcohol-BC association by individual sex hormones. However, a hormonal signature, reflecting lower levels of SHBG and higher levels of sex steroids, mediated a substantial proportion of the association.

Keywords: sex steroids, alcohol, breast cancer, mediation analysis, hormonal signature, EPIC.

This article is protected by copyright. All rights reserved. 


\section{Introduction}

Breast cancer (BC) is the most frequent type of cancer accounting for nearly a quarter of all cancers in women worldwide with about 2.08 million incident breast cancer cases diagnosed in 2018 (1). BC incidence is expected to continue rising with increases in obesity, reductions in fertility and aging of the population, in particular in developing countries (2). BC is a multifactorial disease and its aetiology includes dietary, lifestyle, hormonal, and reproductive risk factors (3). Among these, alcohol intake has been consistently associated with higher BC risk and has been classified by the International Agency for Research on Cancer (IARC) as a carcinogen (Group 1) (4). The evidence is considered strong both in pre- and post-menopausal women (5-8), as confirmed by in a comprehensive analysis by the World Cancer Research Fund (WCRF) (9).

A positive dose-response association between alcohol intake and BC risk, consistently across hormonal receptor status, was shown in a study based on 11,576 incident BC cases within the European Prospective Investigation into Cancer and nutrition (EPIC) cohort (10). Little is known on the mechanisms through which alcohol exerts its carcinogenic effect during BC development, yet accumulating evidence suggests that the association between alcohol intake and breast carcinogenesis may be partly mediated through endogenous sex steroids (11-15). Estrogens and androgens are well-known activators of cellular proliferation, and are associated with an increased BC risk (15). Findings from the EPIC study and the Endogenous Hormones and Breast Cancer Collaborative Group supported the association between elevated pre-diagnostic serum concentrations of oestrogens, androgens and low serum levels of sex hormone binding globulin (SHBG) and higher postmenopausal BC risk 
(16-18). It has been suggested that alcohol consumption increases the concentrations of sex steroids in serum in both pre- and post-menopausal women (15). In EPIC, higher concentrations of androgens including testosterone and free testosterone, and lower concentrations of SHBG were observed in postmenopausal women who consumed more than 25 g/day of alcohol (i.e. 2 glasses) compared with women who were non consumers (19). A review suggested that estrogens could mediate the relationship between alcohol and $\mathrm{BC}$ as alcohol elevates concentrations of circulating oestrogens (15). In postmenopausal women, nearly $100 \%$ of estrogens are synthetized from aromatization of androgens in peripheral tissues, with SHBG regulating their circulating concentrations and that of their free fractions (20).

To our knowledge, only one study conducted within the Women's Health Initiative has explored a causal pathway from alcohol to postmenopausal BC operating through serum estrogen, but no significant evidence was found(21).

Here, we examine whether estradiol, testosterone, their free fractions, and SHBG, as well as a composite hormonal signature, mediated the relationship between alcohol intake and postmenopausal BC in a nested-case control study within EPIC, among alcohol drinkers.

\section{Material and Methods}

The EPIC study. EPIC is a multicentre prospective cohort designed to investigate the associations of diet, lifestyle, environmental and metabolic factors with cancer and other disease outcomes. Over 360,000 women and 150,000 men aged 20-85 years were recruited between 1992 and 2000 from 23 centres spanning 10 European countries: Denmark, France, 
Germany, Greece, Italy, Norway, Spain, Sweden, The Netherlands and the United Kingdom (22). The rationale, study design and methods of the EPIC study have been extensively described (22-24). Biological samples were collected at recruitment prior to disease onset in approximately $80 \%$ of the cohort and were stored at IARC (Lyon, France) in $-196^{\circ} \mathrm{C}$ liquid nitrogen for all countries, except from Denmark (nitrogen vapour, $-150^{\circ} \mathrm{C}$ ) and Sweden (freezers, $-80^{\circ} \mathrm{C}$ ) where samples were stored locally. All participants gave their written informed consent to use their questionnaire data and biological samples for future analyses.

Exposure assessment. During the enrolment period, information on socio-demographic characteristics including education, occupational and recreational levels of physical activity, tobacco smoking, medical and reproductive history, exogenous hormone use, anthropometric measures as well as alcohol consumption habits were gathered using lifestyle questionnaires. Dietary intake over the 12 months was assessed at baseline using validated country-specific dietary questionnaires (self-administered, food frequency questionnaires, semi-quantitative or interviewer-performed) designed to specifically capture local habits with high compliance as detailed elsewhere (22-24). Baseline alcohol intake was calculated from the number of glasses of beer and/or wine, cider, sweet liquors, fortified wines, distilled spirits consumed per day or week in the year preceding recruitment. The individual average daily alcohol intake, expressed in grams per day $(\mathrm{g} / \mathrm{d})$, was computed based on the standard glass volume and ethanol content as the sum of the ethanol content of all alcoholic beverages consumed obtained through country-specific food composition tables per alcoholic beverage type. This calculation was done based on data collected through 24-hr dietary recalls from a subgroup of 
the cohort containing detailed information on alcohol intake distribution during the day in relation to main meals $(25,26)$.

Ascertainment of cancer outcome. Incident BC cases were identified through record linkage with regional cancer and pathology registries with the exception of Naples (Italy), Germany, Greece and France where a combination of methods was employed including: cancer and pathology registries, health insurance records, active follow-up through direct contact with study subjects or next of kin, and collection of clinical records. Vital status was ascertained from municipal, regional or national-level mortality registries. For this study, the closure date was the last date of complete follow-up, both for cancer incidence and vital status, ranging from 2003 to 2006, depending on each EPIC study centre (16,22,27-29). All the self-reported BC cases were systematically verified from clinical and pathologic records. Cancer incidence data were classified according to the International Classification of Diseases for Oncology (ICD-O), as first primary invasive BC, ICD-O codes C50. Information on hormone receptor status (estrogen and progesterone) as well as the laboratory methods and quantification descriptions used to determine the receptor status, were collected by the EPIC centres and criteria were retained to harmonize positive receptor identification across centres (28).

The nested case-control study. The current study is based upon data available from two nested case-control studies within EPIC on postmenopausal BC risk and endogenous hormone levels ("study phase 1" (27) and "study phase 2" (28)). Norway and Sweden were not included in these analyses either because a blood serum sample was not available or because independent studies were being completed on BC risk. In both study phases, postmenopausal women were included. Postmenopausal women were defined as women who had no menstruations in the 
12 months preceding study enrolment, or were older than 55 years of age if the menstrual cycle information was not available, or who had undergone a bilateral oophorectomy. Only women with available blood samples who were not using any menopausal hormone therapy at the time of blood collection (as the use of exogenous hormones influences the endogenous concentrations and some may be the same as endogenous), and who did not have any prevalent cancer at baseline (with the exception of non-melanoma skin cancer) were included into the study. For each case, up to two controls with a blood sample available were chosen at random among appropriate risk sets consisting of all postmenopausal cohort members alive and free of cancer at the time of diagnosis of the case. This was done using an incidence density sampling protocol allowing the inclusion of subjects who became a case later in time, while each control could be sampled more than once (28). Controls were matched to the cases on study recruitment centre, age at blood donation ( \pm 6 months), follow-up time since blood donation ( \pm 3 months), time of the day of blood collection ( \pm 1 hour), and fasting status $(<3$ hours, 3-6 hours, >6 hours). Analyses were also conducted stratifying on estrogen receptor (ER) status. Over the two study phases, there were 798 BC and 1294 matched controls with 387 cases being oestrogen receptor positive (ER+) tumours, 153 oestrogen negative tumours (ER-) and 258 with unknown hormonal receptor status (estrogen and/or progesterone). After excluding case-sets in which the case or her control(s) were non-drinkers (daily intake $<0.1$ /day), the final study sample included 430 cases and 645 matched controls with 218 ER+ (62\% from “study phase 1" and 38\% from “study phase 2”), 105 ER- (27\% from "study phase 1" and 73\% from "study phase 2") and 107 with unknown hormonal receptor status (estrogen and/or progesterone). The ethical review boards of the participating 
institutions/countries/study centres and the International Agency for Research on Cancer (IARC) approved each of the two phases of the study.

Hormone concentrations. For all women in "study phase 1", hormone measurements of estradiol (pmol/L), testosterone (nmol/L) and SHBG (nmol/L) were performed at IARC, while for "study phase 2" they were performed at the German Cancer Research Center (DKFZ). The same assay methods were used whenever possible in the two phases of the study, as detailed elsewhere $(16,28)$. Cases and their matched controls were analysed within the same analytical batch and laboratory technicians were blinded to the case-control status of the study participants. Serum concentrations of free estradiol (pmol/L) and free testosterone (nmol/L) were computed from mass action law equations using absolute concentrations of each sex steroid and SHBG assuming a constant concentration of $43 \mathrm{~g} / \mathrm{L}$ for albumin(28,30,31).

Statistical analyses. In all analyses, baseline alcohol intake (g/day), sex hormones and SHBG levels were log-transformed to normalize their distributions $(\ln ($ alcohol+1)). In addition, for the sex hormones and SHBG, residuals on centre were computed to account for variability that lie in phase of study, distribution across batches of each sex steroid, and the differences of study protocols for sample collection and preparation including treatment and sample handling e.g. thaw-freeze cycles. The residuals were calculated for each biomarker in univariate linear regression models. Geometric means for sex steroids and SHBG in alcohol consumers as well as $2.5^{\text {th }}$ and $97.5^{\text {th }}$ quantiles were computed for cases and controls by study phase. The difference in hormone and SHBG levels between cases and controls was assessed through two-sample t-tests computed on the log-transformed concentrations. 
Partial Least Square (PLS) analysis. With the aim of deriving a hormonal signature associated with alcohol intake, we applied PLS analysis, a multivariate dimension-reduction method that generalises features of principal component analysis with those of multiple linear regression (32). The mathematical and computational details of the PLS method have been thoroughly described in our previous studies within EPIC $(33,34)$. In brief, one PLS factor was retained after performing PLS analysis only amongst controls in the subset of alcohol consumers and a linear combination of the response variables (i.e. estradiol, testosterone and SHBG), was extracted that had maximum covariance with the predictor variable (alcohol intake). Using the loadings derived from the analysis, i.e. the coefficients quantifying the contribution of each hormone to the PLS factor, the PLS score was computed and subsequently extrapolated to the cases. The score was tested as a composite mediator in the alcohol-BC association using mediation analysis as described below. Similarly, a PLS sensitivity analysis was performed including free fractions of estradiol and testosterone from the response set as both these free fractions were computed from SHBG and estradiol or testosterone. The PLS score was calculated as formerly detailed and successively used in a mediation analysis. As the results were virtually unchanged, we did not report the results for this sensitivity analysis.

Alcohol and BC association. The association between alcohol intake and BC risk was first evaluated in multivariable conditional logistic regression models within the total nested casecontrol study. Since no statistically significant association was found for a 1-SD higher logtransformed alcohol intake and risk of overall BC, ER+ and ER- tumours (Table 2), the investigation was restricted to the case-sets of alcohol consumers. 
Mediation analysis. The mediating role of each sex steroids and SHBG (mediator) in the association between alcohol consumption (exposure) and BC (outcome) was examined and mediating effects were assessed separately for each of the considered mediators, and for their composite signature, among alcohol consumers. Estimates of the natural direct effect (NDE), the natural indirect effect (NIE), the total effect (TE) as well as the effect of the mediator adjusted for alcohol, i.e. the exposure, and for confounding variables were computed using a counterfactual approach adapted to dichotomous outcomes (35). Formulae from VanderWeele and Vansteelandt(36) were adapted to accommodate continuous exposures and use of conditional logistic regression models for our matched study design. In brief, two models were specified to obtain NDE and NIE and the odds ratio for the mediator effect adjusted for the exposure. In the outcome model, the exposure and the mediator were related to the $\mathrm{BC}$ indicator in a conditional logistic regression. In models for each mediator of interest, the mediator was linearly regressed on the exposure. This was done only on the subset of controls to account for the nested case-control design (37). The total effect was obtained from a conditional logistic regression relating alcohol intake to BC risk. The formulae detailing how to obtain estimates and their associated 95\%CI and p-value as well as the notations used have been extensively detailed in our previous work (33). Assuming the outcome was rare, we computed the proportion mediated which is measure defined on the risk difference scale and captures the importance of the mediating pathway (37). Based on the estimated odds ratios, this quantity was calculated using the following formula: $\frac{N D E *(N I E-1)}{(N D E * N I E-1)}(36)$. Since mediation analysis was applied to the nested case-control study restricted to alcohol consumers, the interpretation of the causal effects is for an increase of one standard deviation in the exposure 
(log transformed alcohol intake) among alcohol consumers. All models were adjusted for a list of potential confounders including body mass index (BMI, continuous), age at menopause (continuous), and the following categorical variables: smoking status (never, former, current, unknown), education level (none, primary school, technical/professional school, secondary school, longer education including university degree, unknown/unspecified), physical activity index (inactive, moderately inactive, moderately active, active, unknown), use of menopausal hormone therapy (ever vs. never), use of contraceptive pill (ever vs. never), age at first fullterm pregnancy (nulliparous, $<23$ years, 24-25 years, 26-28 years, $>29$ years), number of full term pregnancies (nulliparous, 1 full-term birth, 2 full-term births, 3 full-term births, 4 or more full-term births), and age at menarche ( $<12$ years, 12 years, 13 years, 14 years, $>14$ years). The mediator model was additionally adjusted for study phase (phase 1 vs. phase 2). Interactions between the exposure and each of the confounders were tested both in the outcome model and in the mediator model among controls; with an additional test for exposure-mediator interaction in the former and a term testing interaction between exposure and age at blood collection in the latter. None of the interactions were statistically significant and therefore were not included the final mediation analyses.

All statistical tests were two-sided, p-values below 0.05 were considered statistically significant. All analyses were performed using the R statistical software, with the package 'plsgenomics' used for PLS analysis and mediation computed with an in-house macro.

\section{Results}

The study population characteristics by case-control status are presented in Table $\mathbf{1}$ for the case-sets of alcohol consumers that were examined in this study. Overall, cases had a higher 
average alcohol intake compared with controls (11.3 vs. 9.5g/day) and a higher total energy intake (1970 vs. $1919 \mathrm{kcal} / \mathrm{day})$. Supplemental Table 1 shows the characteristics of the whole population of the nested case-control study. Hormone concentration levels for the study population at baseline restricted to drinker case-sets are shown in Supplemental Table 2 by study phase. Concentrations of testosterone and free testosterone were significantly higher in cases than in controls in "study phase 2", whereas concentrations of estradiol and its free fraction were significantly higher in cases than in controls in “study phase 1”. Additionally, the concentration values for estradiol were on average higher in "study phase 1 " than in “study phase 2” (respectively 99.1 and 89.2 for cases and controls vs. 45.0 and 41.7) likely due to differences in assays between phases.

In our final study population, alcohol intake was statistically significantly associated with higher BC risk with TE OR=1.17(1.01,1.35) for a 1-SD higher log-transformed alcohol intake (Table 2). The association was stronger in ER+ tumours with TE OR=1.36(1.08,1.70, n cases=218). There was no association found for ER- BC $(\mathrm{TE}$ OR=1.29(0.87, 1.91, n cases=105)). A positive association with BC risk overall and for ER+ BC was observed for 1SD increase in log-transformed hormones with $\mathrm{OR}=1.38(1.12,1.70)$ and $1.83(1.28,2.63)$ for estradiol, 1.32(1.08,1.61) and 1.51(1.10,2.08) for free estradiol and 1.21(1.03,1.41) and 1.45(1.13,1.87) for testosterone, respectively. There was an inverse association between SHBG and ER- BC risk (Supplemental Table 3).

Results from individual mediation analyses are presented in Table 3 with estimates for the direct and indirect effects. In the ER+ subset, the NDE estimates for the direct association of alcohol with BC risk considering estradiol, free estradiol, testosterone, free testosterone 
and SHBG as the mediator, were statistically significant with NDE=1.35(1.07,1.70)

1.34(1.07,1.69), 1.30(1.02,1.64), 1.33(1.05,1.68) and 1.35(1.08,1.70), respectively (Table 3).

None of the NIE estimates were statistically significant suggesting that the four sex steroids and SHBG did not mediate the alcohol and BC association individually (Table 3). However, the NIE was borderline significant for free estradiol $(1.03(1.00,1.06))$ suggesting a weak mediation by free estradiol corresponding to a mediated proportion of $19 \%$.

PLS analysis provided a composite signature of estradiol, testosterone and SHBG, as the first PLS factor, with positive loadings for estradiol (0.007) and testosterone (0.070) and a high negative loading for SHBG (-0.141) (Supplemental Table 4). A 1-SD increase in the PLS score was associated with a higher BC risk with $\mathrm{OR}=1.23(1.05,1.43)$ and statistically significantly mediated the association between alcohol intake and overall BC risk with NIE $=1.04(1.01,1.07)$, accounting for a mediated proportion of $24 \%$ of the total effect (Table 4). For ER+ and ER- BC subtypes, the hormonal signature did not mediate the alcohol-BC association as the NIE, corresponding to a proportion mediated of 12 and 36\%, were not statistically significant. The identified signature was however associated with high ER- risk with OR=1.69(1.03,2.69) (Table 4).

\section{Discussion}

In this study restricted to alcohol consumers, a candidate mechanism of the association between alcohol intake and postmenopausal BC development was investigated with mediation analysis. Overall, there was limited evidence that this association was mediated by individual sex-hormone levels with a weak mediation by free estradiol, however, a composite score summarizing information from the individual hormones and SHBG showed that $24 \%$ of the 
relationship between alcohol and BC risk is mediated by a hormonal signature negatively associated with SHBG and positively related to sex steroids.

Alcohol is an established risk factor for BC $(4,9)$, both in pre- and postmenopausal women (6,38). Evidence from a reanalysis of 53 epidemiological studies suggested that the relative risk of BC increased linearly by 7\% for each additional $10 \mathrm{~g} /$ day intake of alcohol (unit of alcohol as defined by WHO)(39). A dose response association was observed in EPIC, irrespective of beverage type, with a higher risk attaining 25\% (17-35\%) for the highest intakes compared to moderate alcohol use (from 0.1 to 5 g/day) (10).

Despite this, the biological pathways that link alcohol with BC development are not well delineated. Hormones and SHBG are involved in complex biological pathways that regulate a host of metabolic functions $(20,40)$. It had previously been suggested that sex-hormones could be involved in the underlying mechanism of the alcohol and BC association (41-43). Several controlled feeding studies $(44,45)$ and observational studies $(14,17,19,46)$ reported associations between alcohol intake and increased sex-hormone blood concentrations in both pre- and postmenopausal women. Compared with non-drinkers, concentrations of estrone, estradiol, and dehydroepiandrosterone sulfate (DHEAS) were higher in women consuming more than $25 \mathrm{~g}$ /day of alcohol in a cross-sectional study in EPIC(14). Findings of similar magnitude were reported in a study of 1,291 postmenopausal controls from a nested casecontrol study in EPIC (19) with 10-20\% larger levels of testosterone and free testosterone and 15\% lower SHBG concentrations in alcohol consumers compared with non-drinkers. Similar associations were reported in a recent cross-sectional analysis examining BC risk factors

This article is protected by copyright. All rights reserved. 
including alcohol and circulating sex hormones measured in over 6,000 postmenopausal controls from 13 prospective studies (17).

The current study is the second of its kind to explore statistical mediation by sex steroids of the alcohol-BC relation. Previously, the mediating role of estradiol was examined in a case-cohort study with 600 cases (of which 401 ER+ and 163 ER-) in the Women's Health Initiative, where no indirect effect was observed suggesting no evidence for alcohol effect through estradiol, although a significant association between alcohol and BC risk overall and in ER+ tumours in postmenopausal women was reported (21). Our study had similar findings in terms of weak evidence of mediation through estradiol, and a strong association of alcohol intake with ER+ tumours. However, it expanded on the latter study by exploring the mediating role of free estradiol, testosterone, free testosterone and SHBG in addition to a hormonal signature.

Strengths of this analysis include the use of harmonized standardised dietary questionnaires which were used to estimate alcohol at baseline. Further, we developed an alcohol-driven hormonal signature that was associated with BC risk and was robust to exclusion of free fractions of estradiol and testosterone from the PLS analysis. Our analyses focused on alcohol consumers, as baseline alcohol non-drinkers may be more healthconscious, may be former heavy drinkers or participants with underlying disease, thus potentially introducing concerns related to reverse causation and, particularly, exposure misclassification. The BC nested case-control study was relatively large in sample size, as it combined two successive rounds of acquisitions of sex steroids in EPIC.

This article is protected by copyright. All rights reserved. 
Nevertheless, our study had limitations, amongst which the generally low alcohol intake of EPIC women, $\sim 8 \mathrm{~g} /$ day on average with $80 \%$ below $15 \mathrm{~g} / \mathrm{d}(10)$, but also among the participants of the BC nested case-control study 10 g/day, which may limit the generalisability of our findings to populations with different alcohol consumption patterns. Another aspect pertains to a key assumption in mediation analysis, which requires a temporal ordering between exposure, mediator and outcome. In our study alcohol was assessed at baseline at the same time of biological samples’ collection, estimating participants’ alcohol intakes over the 12 months preceding enrolment, and endogenous hormones were measured in a single blood sample from each woman reflecting a limited time-frame. Although alcohol intake measurements indicated relatively high validity (24), and androgens, estrogens and SHBG concentrations in postmenopausal women show good reproducibility over time (4749), both exposure and the mediators examined in this study may be subject to measurement errors. Under non-differential measurement error with a normally distributed mediator, the bias of the NIE is towards the null and if direct and indirect effects are in the same direction, the bias of the NDE is away from the null (50). This may have contributed to an underestimation of the indirect effects and an overestimation of the direct effects in our study, resulting in a lower mediated proportion and possibly partially explaining the lack of mediation observed for sex steroids when examined separately. Lastly, in this study different radioimmunoassays were used to measure estradiol between phase 1 and phase 2 (28). For this reason, estradiol concentrations displayed between-studies variations, which we have tried to account for by adjusting for phase of study in the exposure-mediator models.

\section{Conclusion}


Our findings suggested that alcohol intake was associated with higher postmenopausal BC risk in alcohol consumers, overall and for ER+ tumours, with limited evidence of mediation by sex steroids, when examined individually. However, the hormonal signature mediated about $24 \%$ of the alcohol-BC association, suggesting that any potential mechanism of sexsteroids in the alcohol and BC relationship is likely to involve an interplay of hormones, beyond the action of single hormonal levels. Future replication of these findings is needed, possibly in populations with larger amounts of alcohol intake and larger sample size. Finally, our results suggest that sex hormones play a minor role in mediating the alcohol-BC relation and other, possibly unrecognized, pathways are likely involved.

This article is protected by copyright. All rights reserved. 


\section{References}

1. Bray F, Ferlay J, Soerjomataram I, Siegel RL, Torre LA, Jemal A. Global cancer statistics 2018: GLOBOCAN estimates of incidence and mortality worldwide for 36 cancers in 185 countries. CA Cancer J Clin [Internet]. American Cancer Society; 2018 [cited 2018 Oct 4]; Available from: http://doi.wiley.com/10.3322/caac.21492

2. Ferlay J, Ervik M, Lam F, Colombet M, Mery L, Piñeros M, Znaor A, Soerjomataram I, Bray F. Global Cancer Observatory: Cancer Tomorrow. Lyon, France: International Agency for Research on Cancer. 2018.

3. Anothaisintawee T, Wiratkapun C, Lerdsitthichai P, Kasamesup V, Wongwaisayawan S, Srinakarin J, Hirunpat S, Woodtichartpreecha P, Boonlikit S, Teerawattananon Y, et al. Risk factors of breast cancer: a systematic review and meta-analysis. Asia Pac J Public Health. 2013;25:368-87.

4. Baan R, Straif K, Grosse Y, El Ghissassi F, Bouvard V, Altieri A, Cogliano VJ, WHO International Agency for Research on Cancer Monograph Working Group. Carcinogenicity of alcoholic beverages. Lancet Oncol. 2007;8:292-3.

5. Collaborative Group on Hormonal Factors in Breast Cancer. Alcohol , tobacco and breast cancer - collaborative reanalysis of individual data from 53 epidemiological studies , including 58 , 515 women with breast cancer and 95, 067 women without the disease . Collaborative Group On Hormonal Factors In Breast Canc. Br J Cancer. 2002;87:1234-1245.

6. Allen NE, Beral V, Casabonne D, Kan SW, Reeves GK, Brown A, Green J, Million Women Study Collaborators. Moderate Alcohol Intake and Cancer Incidence in

This article is protected by copyright. All rights reserved. 
Women. JNCI J Natl Cancer Inst [Internet]. 2009 [cited 2018 Jul 17];101:296-305. Available from: http://www.ncbi.nlm.nih.gov/pubmed/19244173

7. Chen WY, Rosner B, Hankinson SE, Colditz GA, Willett WC. Moderate alcohol consumption during adult life, drining patterns, and breast cancer. JAMA. 2011;306:1884-90.

8. Boffetta P, Hashibe M. Alcohol and cancer. Lancet Oncol [Internet]. Elsevier; 2006 [cited 2018 Oct 16];7:149-56. Available from: http://www.ncbi.nlm.nih.gov/pubmed/16455479

9. World Cancer Research Fund/American Institute for Cancer Research. Diet, Nutrition, Physical Activity and Cancer: a Global Perspective A summary of the Third Expert Report [Internet]. 2018. Available from: https://dietandcancerreport.org

10. Romieu I, Scoccianti C, Chajès V, Batlle J De, Biessy C, Dossus L, Baglietto L, Clavel-Chapelon F, Overvad K, Olsen A, et al. Alcohol intake and breast cancer in the European prospective investigation into cancer and nutrition. Int J Cancer. 2015;137:1921-30.

11. Scoccianti C, Lauby-Secretan B, Bello P, Chajes V, Romieu I. Female Breast Cancer and Alcohol Consumption. Am J Prev Med. Elsevier; 2014;46:S16-25.

12. Endogenous Hormones and Breast Cancer Collaborative Group, Key TJ, Appleby PN, Reeves GK, Travis RC, Alberg AJ, Barricarte A, Berrino F, Krogh V, Sieri S, et al. Sex hormones and risk of breast cancer in premenopausal women: a collaborative reanalysis of individual participant data from seven prospective studies. Lancet Oncol [Internet]. 2013 [cited 2018 Jul 2];14:1009-19. Available from:

This article is protected by copyright. All rights reserved. 
http://www.ncbi.nlm.nih.gov/pubmed/23890780

13. Erol A, Ho AM-C, Winham SJ, Karpyak VM. Sex hormones in alcohol consumption: a systematic review of evidence. Addict Biol [Internet]. Wiley/Blackwell (10.1111); 2017 [cited 2018 Jul 2]; Available from: http://doi.wiley.com/10.1111/adb.12589

14. Onland-Moret NC, Peeters PHM, van der Schouw YT, Grobbee DE, van Gils CH. Alcohol and Endogenous Sex Steroid Levels in Postmenopausal Women: A CrossSectional Study. J Clin Endocrinol Metab [Internet]. Oxford University Press; 2005 [cited 2018 Jul 16];90:1414-9. Available from: https://academic.oup.com/jcem/articlelookup/doi/10.1210/jc.2004-0614

15. Travis RC, Key TJ. Oestrogen exposure and breast cancer risk. Breast Cancer Res. 2003;5:239-47.

16. Kaaks R, Tikk K, Sookthai D, Schock H, Johnson T, Tj A, Olsen A, Overvad K, Dossus L, Baglietto L, et al. Premenopausal serum sex hormone levels in relation to breast cancer risk, overall and by hormone receptor status — Results from the EPIC cohort. Int J Cancer. 2014;137:1947-57.

17. Endogenous Hormones and Breast Cancer Collaborative Group EH and BCC, Key TJ, Appleby PN, Reeves GK, Roddam AW, Helzlsouer KJ, Alberg AJ, Rollison DE, Dorgan JF, Brinton LA, et al. Circulating sex hormones and breast cancer risk factors in postmenopausal women: reanalysis of 13 studies. Br J Cancer [Internet]. Nature Publishing Group; 2011 [cited 2018 Jul 2];105:709-22. Available from: http://www.ncbi.nlm.nih.gov/pubmed/21772329

18. The Endogenous Hormones and Breast Cancer Collaborative Group. Endogenous Sex

This article is protected by copyright. All rights reserved. 
Hormones and Breast Cancer in Postmenopausal Women: Reanalysis of Nine Prospective Studies. CancerSpectrum Knowl Environ [Internet]. Oxford University Press; 2002 [cited 2018 Jul 2];94:606-16. Available from:

https://academic.oup.com/jnci/article-lookup/doi/10.1093/jnci/94.8.606

19. Rinaldi S, Peeters PHM, Bezemer ID, Dossus L, Biessy C, Sacerdote C, Berrino F, Panico S, Palli D, Tumino R, et al. Relationship of alcohol intake and sex steroid concentrations in blood in pre- and post-menopausal women : the European Prospective Investigation into Cancer and Nutrition. Cancer Causes Control. 2006;17:1033-43.

20. Labrie F. All sex steroids are made intracellularly in peripheral tissues by the mechanisms of intracrinology after menopause. J Steroid Biochem Mol Biol [Internet]. Pergamon; 2015 [cited 2018 Oct 11];145:133-8. Available from: https://www.sciencedirect.com/science/article/pii/S0960076014001150?via\%3Dihub

21. Hvidtfeldt UA, Gunter MJ, Lange T, Chlebowski RT, Lane D, Farhat GN, Freiberg MS, Keiding N, Lee JS, Prentice R, et al. Quantifying mediating effects of endogenous estrogen and insulin in the relation between obesity, alcohol consumption, and breast cancer. Cancer Epidemiol Biomarkers Prev. 2012;21:1203-12.

22. Riboli E, Kaaks R. The EPIC Project: rationale and study design. European Prospective Investigation into Cancer and Nutrition. Int J Epidemiol. 1997;26 Suppl 1:S6-14.

23. Riboli E, Hunt KJ, Slimani N, Ferrari P, Norat T, Fahey M, Charrondière UR, Hémon B, Casagrande C, Vignat J, et al. European Prospective Investigation into Cancer and Nutrition (EPIC): study populations and data collection. Public Health Nutr.

This article is protected by copyright. All rights reserved. 
2002;5:1113-24.

24. Kaaks R, Slimani N, Riboli E. Pilot Phase Studies on the Accuracy of Dietary Intake Measurements in the EPIC Project : Overall Evaluation of Results. 1997;26:26-36.

25. Sieri S, Agudo A, Kesse E, Klipstein-Grobush K, San-José B, Welch AA, Krogh V, Luben R, Allen N, Overvad K, et al. Patterns of alcohol consumption in 10 European countries participating in the European Prospective Investigation into Cancer and Nutrition ( EPIC ) project. Public Health Nutr. 2002;5:1287-96.

26. Slimani N, Deharveng G, Unwin I, Southgate DAT, Vignat J, Skeie G, Salvini S, Parpinel M, Møller A, Ireland J, et al. The EPIC nutrient database project (ENDB): a first attempt to standardize nutrient databases across the 10 European countries participating in the EPIC study. Eur J Clin Nutr. 2007;61:1037-56.

27. Kaaks R, Rinaldi S, Key TJ, Berrino F, Peeters PHM, Biessy C, Dossus L, Lukanova A, Bingham S, Khaw K-T, et al. Postmenopausal serum androgens, oestrogens and breast cancer risk: the European prospective investigation into cancer and nutrition. Endocr Relat Cancer [Internet]. 2005;12:1071-82. Available from: http://erc.endocrinology-journals.org/cgi/doi/10.1677/erc.1.01038

28. James RE, Lukanova A, Dossus L, Becker S, Rinaldi S, Tjønneland A, Olsen A, Overvad K, Mesrine S, Engel P, et al. Postmenopausal Serum Sex Steroids and Risk of Hormone Receptor - Positive and -Negative Breast Cancer : a Nested Case - Control Study. Cancer Prev Res. 2011;4:1626-36.

29. Bergmann MM, Bussas U, Boeing H. Follow-Up Procedures in EPIC-Germany - Data Quality Aspects. Ann Nutr Metab [Internet]. 1999 [cited 2018 Jun 25];43:225-34.

This article is protected by copyright. All rights reserved. 
Available from: http://www.ncbi.nlm.nih.gov/pubmed/10592371

30. Rinaldi S, Geay A, Déchaud H, Biessy C, Zeleniuch-Jacquotte A, Akhmedkhanov A, Shore RE, Riboli E, Toniolo P, Kaaks R. Validity of free testosterone and free estradiol determinations in serum samples from postmenopausal women by theoretical calculations. Cancer Epidemiol Biomarkers Prev [Internet]. 2002 [cited 2018 Jun 26];11:1065-71. Available from: http://www.ncbi.nlm.nih.gov/pubmed/12376508

31. Vermeulen A, Verdonck L, Kaufman JM. A Critical Evaluation of Simple Methods for the Estimation of Free Testosterone in Serum. J Clin Endocrinol Metab [Internet]. 1999 [cited 2018 Jun 26];84:3666-72. Available from: http://www.ncbi.nlm.nih.gov/pubmed/10523012

32. Tenenhaus M. La régression PLS. Technip. Paris; 1998.

33. Assi N, Thomas DC, Leitzmann M, Stepien M, Chajes V, Philip T, Vineis P, Bamia C, Boutron-Ruault M-C, Sandanger TM, et al. Are metabolic signatures mediating the relationship between lifestyle factors and hepatocellular carcinoma risk? Results from a nested case-control study in EPIC. Cancer Epidemiol Biomarkers Prev. 2018;27.

34. Assi N, Fages A, Vineis P, Chadeau-Hyam M, Stepien M, Duarte-Salles T, Byrnes G, Boumaza H, Knuppel S, Kuhn T, et al. A statistical framework to model the meetingin-the-middle principle using metabolomic data: application to hepatocellular carcinoma in the EPIC study. Mutagenesis [Internet]. 2015;30:743-53. Available from: http://www.ncbi.nlm.nih.gov/pubmed/26130468

35. Valeri L, Vanderweele TJ. Mediation analysis allowing for exposure-mediator interactions and causal interpretation: theoretical assumptions and implementation with

This article is protected by copyright. All rights reserved. 
SAS and SPSS macros. Psychol Methods. 2013;18:137-50.

36. Vanderweele TJ, Vansteelandt S. Odds ratios for mediation analysis for a dichotomous outcome. Am J Epidemiol. 2010;172:1339-48.

37. Vanderweele TJ. Explanation in Causal Inference: Methods for Mediation and Interaction. 1st editio. New York USA: Oxford University Press; 2015. 728 p.

38. Zhang SM, Lee I-M, Manson JE, Cook NR, Willett WC, Buring JE. Alcohol Consumption and Breast Cancer Risk in the Women’s Health Study. Am J Epidemiol [Internet]. 2007 [cited 2018 Jul 17];165:667-76. Available from: http://www.ncbi.nlm.nih.gov/pubmed/17204515

39. Hamajima N, Hirose K, Tajima K, Rohan T, Calle EE, Heath CW, Coates RJ, Liff JM, Talamini R, Chantarakul N, et al. Alcohol, tobacco and breast cancer--collaborative reanalysis of individual data from 53 epidemiological studies, including 58,515 women with breast cancer and 95,067 women without the disease. Br J Cancer [Internet]. Nature Publishing Group; 2002 [cited 2018 Jul 17];87:1234-45. Available from: http://www.ncbi.nlm.nih.gov/pubmed/12439712

40. Kufe DW, Holland JF, Frei E, American Cancer Society. Holland-Frei Cancer medicine, 6th edition. 6th Editio. BC Decker; 2003.

41. Dumitrescu RG, Cotarla I. Understanding breast cancer risk -- where do we stand in 2005? J Cell Mol Med [Internet]. [cited 2018 Jul 17];9:208-21. Available from: http://www.ncbi.nlm.nih.gov/pubmed/15784178

42. Mitrunen K, Hirvonen A. Molecular epidemiology of sporadic breast cancer. The role of polymorphic genes involved in oestrogen biosynthesis and metabolism. Mutat Res

This article is protected by copyright. All rights reserved. 
[Internet]. 2003 [cited 2018 Jul 17];544:9-41. Available from:

http://www.ncbi.nlm.nih.gov/pubmed/12888106

43. Singletary KW, Gapstur SM. Alcohol and Breast Cancer. JAMA [Internet]. American Medical Association; 2001 [cited 2018 Jul 17];286:2143. Available from: http://jama.jamanetwork.com/article.aspx?doi=10.1001/jama.286.17.2143

44. Mahabir S, Baer DJ, Johnson LL, Dorgan JF, Campbell W, Brown E, Hartman TJ, Clevidence B, Albanes D, Judd JT, et al. The effects of moderate alcohol supplementation on estrone sulfate and DHEAS in postmenopausal women in a controlled feeding study. Nutr J [Internet]. 2004 [cited 2018 Jul 18];3:11. Available from: http://www.ncbi.nlm.nih.gov/pubmed/15353002

45. Dorgan JF, Baer DJ, Albert PS, Judd JT, Brown ED, Corle DK, Campbell WS, Hartman TJ, Tejpar AA, Clevidence BA, et al. Serum Hormones and the AlcoholBreast Cancer Association in Postmenopausal Women. J Natl Cancer Inst [Internet]. Oxford University Press; 2001 [cited 2018 Jul 11];93:710-5. Available from: https://academic.oup.com/jnci/article-lookup/doi/10.1093/jnci/93.9.710

46. Kopp TI, Jensen DM, Ravn-Haren G, Cohen A, Sommer HM, Dragsted LO, Tjonneland A, Hougaard DM, Vogel U. Alcohol-related breast cancer in postmenopausal women - effect of CYP19A1, PPARG and PPARGC1A polymorphisms on female sex-hormone levels and interaction with alcohol consumption and NSAID usage in a nested case-control study and a randomised controlled tr. BMC Cancer [Internet]. BioMed Central; 2016 [cited 2018 Jul 9];16:283. Available from: http://bmccancer.biomedcentral.com/articles/10.1186/s12885-016-

This article is protected by copyright. All rights reserved. 
2317-y

47. Zeleniuch-Jacquotte A, Gu Y, Shore RE, Koenig KL, Arslan AA, Kato I, Rinaldi S, Kaaks R, Toniolo P. Postmenopausal levels of sex hormones and risk of breast carcinoma in situ: results of a prospective study. Int J Cancer [Internet]. Wiley-Liss Inc.; 2005 [cited 2018 Jul 24];114:323-7. Available from: http://www.ncbi.nlm.nih.gov/pubmed/15540225

48. Micheli A, Muti P, Pisani P, Secreto G, Recchione C, Totis A, Fissi R, Cavalleri A, Panico S, Berrino F. Repeated serum and urinary androgen measurements in premenopausal and postmenopausal women. J Clin Epidemiol [Internet]. Pergamon; 1991 [cited 2018 Jul 24];44:1055-61. Available from: https://www.sciencedirect.com/science/article/pii/089543569190007V

49. Lukanova A, Lundin E, Micheli A, Arslan A, Ferrari P, Rinaldi S, Krogh V, Lenner P, Shore RE, Biessy C, et al. Circulating levels of sex steroid hormones and risk of endometrial cancer in postmenopausal women. Int J Cancer [Internet]. WileyBlackwell; 2004 [cited 2018 Jul 24];108:425-32. Available from: http://doi.wiley.com/10.1002/ijc.11529

50. VanderWeele TJ, Valeri L, Ogburn EL. The role of measurement error and misclassification in mediation analysis: mediation and measurement error. Epidemiology [Internet]. NIH Public Access; 2012 [cited 2018 Jul 13];23:561-4. Available from: http://www.ncbi.nlm.nih.gov/pubmed/22659547

This article is protected by copyright. All rights reserved. 


\section{Tables}

Table 1: Baseline characteristics of the study population of the EPIC nested case-control study on postmenopausal BC casesets where both cases and controls are alcohol consumers at baseline (>0.1g/day).

\begin{tabular}{|c|c|c|}
\hline Characteristics $^{*}$ & Cases & Controls \\
\hline Number of subjects & 430 & 645 \\
\hline Casesets with ER positive tumours & 218 & 318 \\
\hline Casesets with ER negative tumours & 105 & 126 \\
\hline Age at blood collection (y) & $59.9(50.5,71.3)$ & $60.1(50.9,71.4)$ \\
\hline Height $(\mathrm{cm})$ & $162.0(150.5,174.6)$ & $161.4(149.1,174.0)$ \\
\hline Weight (kg) & $69.0(50.7,98.4)$ & $66.6(49.2,90.5)$ \\
\hline BMI (kg/m2) & $26.3(19.7,37.1)$ & $25.6(19.3,35.5)$ \\
\hline Total energy intake (kcal/day) & $1970.4(1117.6,3063.4)$ & $1919.1(1077.7,3095.9)$ \\
\hline Alcohol intake at recruitment (g/day) & $11.3(0.20,44.9)$ & $9.5(0.20,42.5)$ \\
\hline Age at menopause (y) & $49.3(38.0,57.0)$ & $48.9(36.2,56.9)$ \\
\hline Years between blood donation and diagnosis (y) & $3.7(0.6,9.5)$ & - \\
\hline \multicolumn{3}{|l|}{ Age at menarche } \\
\hline$<12$ years & $54(12.6)$ & $69(10.7)$ \\
\hline 12 years & 85 (18.9) & $128(19.8)$ \\
\hline 13 years & $91(21.2)$ & $140(21.7)$ \\
\hline 14 years & $104(24.2)$ & $153(23.7)$ \\
\hline$>14$ years & $86(20.0)$ & $145(22.5)$ \\
\hline Unknown & $10(2.3)$ & $10(1.6)$ \\
\hline \multicolumn{3}{|l|}{ Age at first full term pregnancy } \\
\hline Nulliparous & $69(16.0)$ & $88(13.6)$ \\
\hline$<23$ years & $118(27.4)$ & $198(30.7)$ \\
\hline 24-25 years & $62(14.4)$ & $115(17.8)$ \\
\hline $26-28$ years & $96(22.3)$ & $141(21.9)$ \\
\hline$>29$ years & $85(19.8)$ & $103(16.0)$ \\
\hline \multicolumn{3}{|l|}{ Use of contraceptive pill ${ }^{* *}$} \\
\hline Ever & $175(40.7)$ & $269(41.7)$ \\
\hline Never & $248(57.7)$ & $374(58.0)$ \\
\hline Unknown & $7(1.6)$ & $2(0.3)$ \\
\hline \multicolumn{3}{|l|}{ Use of hormonal menopause therapy ${ }^{* *}$} \\
\hline 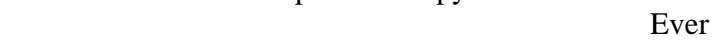 & $87(20.2)$ & $142(22.0)$ \\
\hline Never & $342(79.6)$ & $502(77.8)$ \\
\hline Unknown & $1(0.2)$ & $1(0.2)$ \\
\hline \multicolumn{3}{|l|}{ Physical activity levels } \\
\hline Active & $94(21.9)$ & $134(20.8)$ \\
\hline Moderately active & $71(16.5)$ & $123(19.1)$ \\
\hline Moderately inactive & $162(37.7)$ & $221(34.3)$ \\
\hline Inactive & $102(23.7)$ & $164(25.4)$ \\
\hline Unknown & $1(0.2)$ & $3(0.5)$ \\
\hline \multicolumn{3}{|l|}{ Smoking Status } \\
\hline Never & $241(56.0)$ & 345 (53.5) \\
\hline Former & $116(27.0)$ & $175(27.1)$ \\
\hline Current smoker & $71(16.5)$ & $121(18.8)$ \\
\hline Unknown & $2(0.5)$ & $4(0.6)$ \\
\hline \multicolumn{3}{|l|}{ Fasting status at the time of blood collection } \\
\hline No $(<3 h)$ & $240(55.8)$ & 360 (55.8) \\
\hline In between (3-6h) & $86(20.0)$ & $116(18.0)$ \\
\hline Yes (>6h) & $92(21.4)$ & 145 (22.5) \\
\hline Unknown & $12(2.8)$ & $24(3.7)$ \\
\hline \multicolumn{3}{|l|}{ Education level } \\
\hline None & $4(0.9)$ & $9(1.4)$ \\
\hline Primary school completed & $158(36.7)$ & 238 (36.9) \\
\hline Secondary school & $69(16.0)$ & $107(16.6)$ \\
\hline Technical / Professional school & $110(25.6)$ & 167 (25.9) \\
\hline
\end{tabular}


${ }^{*}$ Values are presented as means and 2.5th and 97.5th percentiles in parentheses for continuous variables and as frequencies and percentages in parentheses for categorical variables. ${ }^{* *}$ Women included in the study were not using any form of exogenous hormones at recruitment. 
Table 2: Odds ratio and 95\% confidence intervals (95\%CI) for the Total Effects (TE) of alcohol on postmenopausal BC for 1-SD increase in log-alcohol intake.

\begin{tabular}{lcl}
\hline Tumour type & N cases/ N controls & TE $\mathbf{( 9 5 \% C I ) *}$ \\
\hline On total nested case-control study & \\
ER positive & $798 / 1294$ & $1.03(0.93,1.14)$ \\
ER negative & $387 / 612$ & $1.04(0.89,1.21)$ \\
\hline In nested case-control study restricted to alcohol consumers case-sets $(>0.1 \mathrm{~g} /$ day $)$ \\
\hline Overall BC & $153 / 193$ & $\mathbf{1 . 1 7}(\mathbf{1 . 0 1 , 1 . 3 5})$ \\
ER positive & $430 / 645$ & $\mathbf{1 . 3 6}(\mathbf{1 . 0 8 , 1 . 7 0 )}$ \\
ER negative & $218 / 318$ & $1.29(0.87,1.91)$ \\
\hline
\end{tabular}

*Statistically significant TE are displayed in bold font. 
Table 3: Results from the mediation analyses in case-sets of alcohol consumers ( $>0.1 \mathrm{~g} / \mathrm{day})$, with ORs ${ }^{*}$ and their associated 95\% CIs for the Natural Direct Effect (NDE) and the Natural Indirect Effect (NIE) using residuals based on Centre for the log-transformed hormone levels.

\begin{tabular}{|c|c|c|c|}
\hline Hormone & NDE (95\%CI) & NIE (95\%CI) & \% mediated - RD scale ${ }^{\dagger}$ \\
\hline \multicolumn{4}{|c|}{ Overall BC } \\
\hline Estradiol & $1.15(1.00,1.33)$ & $1.02(0.99,1.04)$ & 13 \\
\hline Free Estradiol & $1.15(0.99,1.33)$ & $1.03(1.00,1.06)$ & 19 \\
\hline Testosterone & $1.12(0.96,1.30)$ & $1.02(1.00,1.04)$ & 16 \\
\hline Free Testosterone & $1.13(0.98,1.31)$ & $1.01(0.99,1.03)$ & 8 \\
\hline SHBG & $1.16(1.00,1.34)$ & $1.02(0.99,1.05)$ & 13 \\
\hline \multicolumn{4}{|c|}{ ER positive } \\
\hline Estradiol & $1.35(1.07,1.70)$ & $1.06(0.98,1.14)$ & 19 \\
\hline Free Estradiol & $1.34(1.07,1.69)$ & $1.06(0.99,1.13)$ & 19 \\
\hline Testosterone & $1.30(1.02,1.64)$ & $1.00(0.95,1.05)$ & 0 \\
\hline Free Testosterone & $1.33(1.05,1.68)$ & $1.00(0.98,1.01)$ & 0 \\
\hline SHBG & $1.35(1.08,1.70)$ & $1.00(0.96,1.05)$ & 0 \\
\hline \multicolumn{4}{|c|}{ ER negative } \\
\hline Estradiol & $1.31(0.88,1.95)$ & $1.01(0.97,1.06)$ & 4 \\
\hline Free Estradiol & $1.31(0.86,2.00)$ & $1.03(0.95,1.10)$ & 11 \\
\hline Testosterone & $1.25(0.83,1.87)$ & $1.01(0.98,1.04)$ & 5 \\
\hline Free Testosterone & $1.18(0.76,1.82)$ & $1.04(0.96,1.13)$ & 21 \\
\hline SHBG & $1.19(0.77,1.84)$ & $1.08(0.97,1.22)$ & 33 \\
\hline
\end{tabular}

* In the mediation analysis, the exposure was the log-transformed alcohol at baseline, the mediator was in turn each one of the log-transformed hormones (residuals on Centre), and the outcome was postmenopausal BC (subtypes listed above).The outcome models were computed through conditional logistic regressions. The mediator models were linear and additionally adjusted for phase of study. All models were adjusted for BMI (continuous), age at menopause (cont.), smoking status (categorical), education level (cat.), physical activity index (cat.), use of exogenous hormones (ever vs. never), use of pill (ever vs. never), number of full term pregnancies (cat.), age at full term pregnancy (cat.) and age at menarche (cat.). Cases and controls were matched on study recruitment centre, age at blood collection ( $\pm 6 \mathrm{months})$, time of the day at blood collection ( \pm 1 hour), fasting status ( $<3 \mathrm{~h}, 3-6 \mathrm{~h},>6 \mathrm{~h})$ and study phase (1 or 2$)$.

${ }^{\dagger}$ NDE and NIE, their 95\% CIs and proportion mediated on the risk difference (RD) scale are computed from formulae as detailed in Materials and Methods. ORs are expressed for an increase in one standard deviation of the residuals on Centre of the log-transformed hormone variable. The NDE and NIE are expressed for an increase in one standard deviation of the log-transformed alcohol intake.

Bold font indicating statistically significant findings. 
Table 4: Results from the mediation analyses: OR for the association between the PLS factor and postmenopausal BC and NDE and NIE and their respective 95\%CI.

\begin{tabular}{|c|c|c|c|c|}
\hline & OR (95\%CI) & NDE (95\%CI) & NIE (95\%CI) & $\begin{array}{l}\text { \% mediated } \\
\text { - RD scale }\end{array}$ \\
\hline Overall BC & $1.23(1.05,1.43)$ & $1.14(0.98,1.32)$ & $1.04(1.01,1.07)$ & 24 \\
\hline ER positive & $1.20(0.95,1.51)$ & $1.33(1.06,1.67)$ & $1.04(0.99,1.09)$ & 12 \\
\hline ER negative & $1.67(1.03,2.69)$ & $1.15(0.75,1.75)$ & $1.07(0.97,1.19)$ & 36 \\
\hline
\end{tabular}

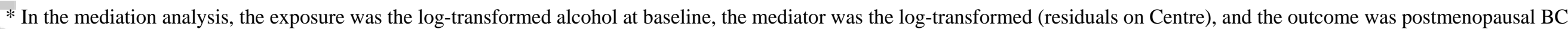

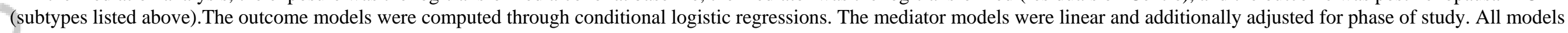

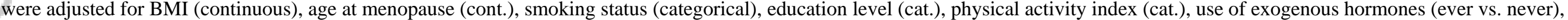

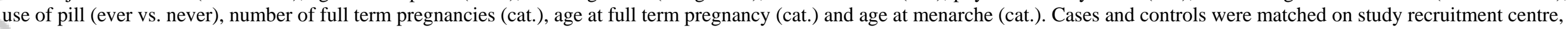
age at blood collection ( \pm 6 months), time of the day at blood collection ( \pm 1 hour), fasting status ( $<3 \mathrm{~h}, 3-6 \mathrm{~h},>6 \mathrm{~h})$ and study phase $(1$ or 2$)$.

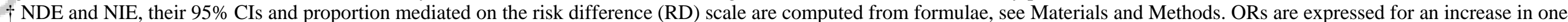
standard deviation of the PLS hormonal signature score. The NDE and NIE are expressed for an increase in one standard deviation of the log-transformed alcohol intake.

Bold font indicating statistically significant findings. 


\section{University Library}

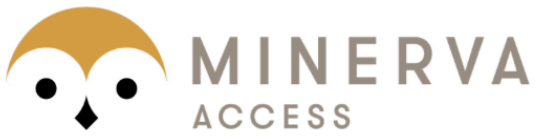

A gateway to Melbourne's research publications

Minerva Access is the Institutional Repository of The University of Melbourne

\section{Author/s:}

Assi, N;Rinaldi, S;Viallon, V;Dashti, SG;Dossus, L;Fournier, A;Cervenka, I;Kvaskoff, M;Turzanski-Fortner, R;Bergmann, M;Boeing, H;Panico, S;Ricceri, F;Palli, D;Tumino, R;Grioni, S;Sanchez Perez, MJ;Chirlaque, M-D;Bonet, C;Barricarte Gurrea, A;Amiano Etxezarreta, P;Merino, S; de Mesquita, HBB;van Gils, CH;Onland-Moret, C;Tjonneland, A;Overvad, K;Trichopoulou, A;Martimianaki, G;Karakatsani, A;Key, T;Christakoudi, S;Ellingjord-Dale, M;Tsilidis, K;Riboli, E;Kaaks, R;Gunter, MJ;Ferrari, P

Title:

Mediation analysis of the alcohol-postmenopausal breast cancer relationship by sex hormones in the EPIC cohort

\section{Date:}

2020-02-01

\section{Citation:}

Assi, N., Rinaldi, S., Viallon, V., Dashti, S. G., Dossus, L., Fournier, A., Cervenka, I., Kvaskoff, M., Turzanski-Fortner, R., Bergmann, M., Boeing, H., Panico, S., Ricceri, F., Palli, D., Tumino, R., Grioni, S., Sanchez Perez, M. J., Chirlaque, M. -D., Bonet, C. ,... Ferrari, P. (2020). Mediation analysis of the alcohol-postmenopausal breast cancer relationship by sex hormones in the EPIC cohort. INTERNATIONAL JOURNAL OF CANCER, 146 (3), pp.759-768. https://doi.org/10.1002/ijc.32324.

Persistent Link:

http://hdl.handle.net/11343/285799 